\title{
Bias asymmetry in the conductance profile of magnetic ions on surfaces probed by scanning tunneling microscopy
}

\author{
Aaron Hurley, Nadjib Baadji, and Stefano Sanvito \\ School of Physics and CRANN, Trinity College, Dublin 2, Ireland
}

(Received 29 March 2012; published 7 September 2012)

\begin{abstract}
The conductance profiles of magnetic transition-metal atoms, such as $\mathrm{Fe}$, $\mathrm{Co}$, and $\mathrm{Mn}$, deposited on surfaces and probed by a scanning tunneling microscope (STM), provide detailed information on the magnetic excitations of such nanomagnets. In general, the profiles are symmetric with respect to the applied bias. However, a set of recent experiments has shown evidence for inherent asymmetries when either a normal or a spin-polarized STM tip is used. In order to explain such asymmetries, here we expand our previously developed perturbative approach to electron-spin scattering to the spin-polarized case and to the inclusion of out of equilibrium spin populations. In the case of a magnetic STM tip, we demonstrate that the asymmetries are driven by the nonequilibrium occupation of the various atomic spin levels, an effect that is reminiscent of electron spin transfer. In contrast, when the tip is not spin polarized, such a nonequilibrium population cannot be built up. In this circumstance, we propose that the asymmetry simply originates from the transition metal ion density of states and from the uneven electronic coupling between the scattering magnetic atoms and both the tip and the substrate. These effects are included in the formalism as a nonvanishing real component to the spin-scattering self-energy.
\end{abstract}

DOI: 10.1103/PhysRevB.86.125411

PACS number(s): 72.15.Qm, 71.10.-w, 73.40.Gk

\section{INTRODUCTION}

The possibility of altering and controlling the spin-state of a single magnetic ion or of a small magnetic cluster with an external probe represents a unique opportunity towards the understanding and the exploitation of the magnetic interaction at the nanoscale level. Possible areas of application for such an ability may include spin-based quantum logic, where one necessitates to prepare, manipulate, and read spin qubits. It is then crucial to develop tools capable of addressing the single spin limit. Low-temperature scanning tunneling microscopy provides one such tool. In general, the method exploits a scanning tunneling microscope (STM) operated in spectroscopical mode by which the inelastic electron tunneling spectroscopy (IETS) at the spin excitations of a given system is measured. ${ }^{1}$ This scheme is known as spin-flip IETS (SF-IETS). The same STM can also be used to position and manipulate the magnetic atoms on a nonmagnetic substrate, ${ }^{2}$ so that STM appears both as a fabrication and subtle characterization tool.

Transition-metal magnetic atoms on insulating surfaces, in particular, $\mathrm{Mn},{ }^{3,4} \mathrm{Co},{ }^{5,6}$ and $\mathrm{Fe},{ }^{7-9}$ have been the focus of intensive research in the last few years. These have all partially filled $d$ shells, which are highly localized and responsible for the magnetic moment, and extended $s$-like electrons, which are responsible for the electron conduction. In general, $s$ and $d$ electrons interact via exchange coupling so that the magnetic structure is coupled to the conducting electrons. The magnetic atoms are usually deposited on carefully prepared $\mathrm{CuN}$-decorated $\mathrm{Cu}$ surfaces, where the typical electronic coupling is weak enough that the magnetism is preserved, but it is sufficiently strong to break the atomic central symmetry so that magnetic anisotropy develops. STM experiments are then conducted and the fingerprint of a magnetic excitation is a step in the differential conductance, $G=d I / d V$, as a function of bias, $V$ ( $I$ is the STM current). These appear at the critical voltage necessary to open a new inelastic transport channel, i.e., at voltages corresponding to the given magnetic excitation energy.
Several methods aimed at modeling SF-IETS have been recently developed. Early theoretical work has focused on second-order perturbation theory to describe the experimental conductance spectra of equilibrium spins by either using a master equation approach ${ }^{10-15}$ or a nonequilibrium Green's function one. ${ }^{16}$ More recently, this scheme has been extended to third order, which allows us to describe additional features in the $G(V)$ line shape that cannot be accounted for at the second-order level. ${ }^{17-20}$ These works have been very successful in describing conductance profiles, which appear symmetric with the external bias polarity, i.e., that they cannot distinguish whether the current flows from the sample to the tip or in the opposite direction. However, recent experiments have shown that regardless of whether a non-spin-polarized ${ }^{3}$ or a spin-polarized ${ }^{4,9}$ tip is used, the IETS profiles exhibit an intrinsic asymmetry with respect to the applied voltage, i.e., $G(V) \neq G(-V)$.

In the case of a spin-polarized STM tip, where the tip density of states is spin split between majority (spin up) and minority (spin down) carriers, the asymmetry has been theoretically well explained., ${ }^{4,21-24}$ It has been shown that spin selection rules enforce a suppression of the inelastic scattering, which depends on the direction of the electrons flow. This results in an asymmetric conductance profile, where the magnitude of the asymmetry depends directly on the spin polarization of the tip. It is also well understood that by driving spins out of equilibrium (e.g., by decreasing the tip-sample distance) the conductance line shape changes. ${ }^{4,9,22,23,25}$ In this case, we must assume that the tunneling electrons influence the spin state of the atom as the time between inelastic events is small compared to the spin relaxation time. A tunneling electron can then encounter the local spin in an excited state far from the ground state. The nonequilibrium population of the various accessible spin states then becomes bias dependent and, for spin-polarized tips, this enhances the asymmetry of the $G(V)$ line shape.

Also in the case of a non-spin-polarized tip, a bias asymmetry has been revealed experimentally. ${ }^{7}$ In particular, 
this appears to be quite prominent for both single Mn atoms and Mn mono-atomic chains. This feature has been previously ascribed to a shift in the magnetic atom on-site energy, i.e., to an effect arising from the details of the density of states of the atom producing scattering. Such a density-of-states effect produces a nontrivial slope in the conductance as a function of bias. ${ }^{26}$ The on-site energy shift, however, does not account for the asymmetry seen in the inelastic step heights, which also depends on bias and on the ratio between tip and substrate coupling to the sample. ${ }^{27,28}$ Here, we provide an alternative theoretical description, which allows us to better fit the experimentally found conductance line shape.

In our previous works, ${ }^{16,17}$ we have combined the nonequilibrium Green's function (NEGF) ${ }^{29,30}$ formalism with a perturbative expansion of the electron-spin interaction in order to describe SF-IETS spectra in a manner that is fully amenable to an implementation within density functional theory (DFT). ${ }^{31,32}$ The scheme essentially consists in constructing an electron-spin interaction self-energy, which describes the inelastic tunneling events. The interacting self-energy was previously expanded first up to second order ${ }^{16}$ and then to the third order, ${ }^{17}$ with this latter describing the logarithmic decays of $G(V)$ at each conductance step. Although both nonequilibrium effects and spin-polarized IETS have been well described up to the second order by the master equation approach, in this work, we extend our formalism to include the extra line-shape features that can be ascribed to the third-order self-energy. We also propose that the real part of the interacting self-energy, which has been well studied in the case of electron-phonon interactions, ${ }^{33,34}$ is a necessary addition to the NEGF formalism in order to account for asymmetric features in nonspin polarized systems.

The layout of the paper is as follows. In the next section, we extend our NEGF formalism to account for spin-polarized leads. In the same section we also derive a second-order electron-spin self-energy, which includes a second-order expansion of the spin propagator. This provides the means to study nonequilibrium effects that result from high current densities. Furthermore, in the case of non-spin-polarized tips, we derive an expression for the real part of the scattering self-energy up to second order. All of the above is combined with the third-order contribution to the electron propagator. We then move to the results. First, we study the nonequilibrium effects arising from an increase in current density for the case of a Mn dimer. Then we recreate the spin-polarized experiments of Loth et al. on single $\mathrm{Mn}$ and $\mathrm{Fe}$ atoms. ${ }^{4,9}$ Finally, we test how the inclusion of the real part of the scattering self-energy modifies the SF-IETS for a nonpolarized tip probing Mn monomers and trimers.

\section{THEORETICAL MODELS}

\section{A. Interaction Hamiltonian and contour Green's functions}

We consider here the same single-orbital tight-binding model used in our previous works ${ }^{16,17}$ to describe a magnetic system (S) coupled to two noninteracting electrodes representing respectively the STM tip (tip) and the substrate (sub). The scattering region containing the magnetic nanostructure consists of a one-dimensional chain of $N$ magnetic atoms.
Each of the $i$ th atoms carries a quantum mechanical spin $\mathbf{S}_{i}$ and it is characterized by an on-site energy $\varepsilon_{0}$. We assume that the tip and the substrate can only couple to one atom at a time in the scattering region thus to broaden the electronic level $\varepsilon_{0}$ through the interaction with the electrode by $\Gamma_{\text {tip-S/sub-s }}$. The Hamiltonian in the scattering region is then described by $H_{\mathrm{S}}=H_{e}+H_{s p}+H_{e-s p}$, where $H_{\mathrm{e}}$ is the tight-binding electronic part, $H_{s p}$ is the spin part, and $H_{e-s p}$ describes the electron-spin interaction. The various terms can be written explicitly as

$$
\begin{gathered}
H_{s p}=J_{d d} \sum_{\lambda}^{N-1} \mathbf{S}_{\lambda} \cdot \mathbf{S}_{\lambda+1}+\sum_{\lambda}^{N}\left\{g \mu_{\mathrm{B}} \mathbf{B} \cdot \mathbf{S}_{\lambda}\right. \\
\left.+D\left(S_{\lambda}^{z}\right)^{2}+E\left[\left(S_{\lambda}^{x}\right)^{2}-\left(S_{\lambda}^{y}\right)^{2}\right]\right\}, \\
H_{e-s p}=J_{s d} \sum_{\lambda \alpha, \alpha^{\prime}}\left(c_{\lambda \alpha}^{\dagger}[\sigma]_{\alpha \alpha^{\prime}} c_{\lambda \alpha^{\prime}}\right) \cdot \mathbf{S}_{\lambda}+\varepsilon_{0} \sum_{\lambda \alpha} c_{\lambda \alpha}^{\dagger} c_{\lambda \alpha},
\end{gathered}
$$

where the electron ladder operator $c_{i \alpha}^{\dagger}\left(c_{i \alpha}\right)$ creates (annihilates) an electron at site $i$ with $\operatorname{spin} \alpha(\alpha=\uparrow, \downarrow)$ and on-site energy $\varepsilon_{0}$.

We model the spin-spin interaction between the localized $\left\{\mathbf{S}_{i}\right\}$ spins by a nearest-neighbor Heisenberg Hamiltonian with coupling strength $J_{d d}$. Furthermore, we include interaction with an external magnetic field $\mathbf{B}\left(\mu_{\mathrm{B}}\right.$ is the Bohr magneton and $g$ is the gyromagnetic ratio) and both uniaxial and transverse anisotropy of magnitude $D$ and $E$, respectively. ${ }^{7,35}$ The electron-spin interaction Hamiltonian is constructed within the $s$ - $d$ model, ${ }^{36,37}$ where the transport electrons $s$ are locally exchanged coupled to quantum spins $\left\{\mathbf{S}_{i}\right\}$ ( $d$ indicates that the local moments originating from the atomic $d$ shell). In Eq. (2), the interaction strength is $J_{s d}$ and $\sigma$ is a vector of Pauli matrices. The second term in Eq. (2) represents the onsite energy with strength $\epsilon_{0}$. This is included in the electron-spin coupling Hamiltonian because it also generates elastic scattering to the $s-d$ interaction (therefore this term will also be included in the perturbation expansion). The ratio, $\chi=\epsilon_{0} / J_{s d}$, is typically in the range $1-2 .^{22,23}$ This term was not included in our previous works ${ }^{16,17}$ as it only becomes important for spin-polarized electrodes.

In order to construct an electron-spin interacting self-

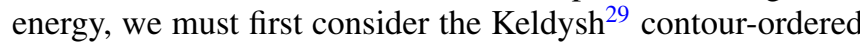
single-body Green's functions (propagators) for both the electronic $(G)$ and the spin $(D)$ subsystems in the electron-spin many-body ground state |\rangle :

$$
\begin{aligned}
& {\left[G\left(\tau, \tau^{\prime}\right)\right]_{\alpha \alpha^{\prime}}=-i\left\langle\left|T_{C}\left\{c_{\alpha}(\tau) c_{\alpha^{\prime}}^{\dagger}\left(\tau^{\prime}\right)\right\}\right|\right\rangle,} \\
& {\left[D\left(\tau, \tau^{\prime}\right)\right]_{n m}=-i\left\langle\left|T_{C}\left\{d_{n}(\tau) d_{m}^{\dagger}\left(\tau^{\prime}\right)\right\}\right|\right\rangle .}
\end{aligned}
$$

These propagators describe a nonequilibrium system at zero temperature. Here, $d_{m}^{\dagger}$ is a quasiparticle creation operator defined by the relation

$$
S^{i}(\tau)=\sum_{m n} S_{m n}^{i} d_{m}^{\dagger}(\tau) d_{n}(\tau),
$$

where $i=\{x, y, z\}$ and $m, n$ are eigenstates of $H_{s p}$ of energy $\varepsilon_{m}, \varepsilon_{n}$. The matrix elements $S_{m n}^{i}=\left\langle m\left|S^{i}\right| n\right\rangle$ determine the transition rates from the initial state $n$ to the final state $m$. The quasiparticle operators are assumed fermionic in nature. ${ }^{16}$ 
Therefore the equilibrium population of a given state $m$ is given by

$$
P_{m}=d_{m}^{\dagger} d_{m}=\frac{1}{\exp \left(\frac{\varepsilon_{m}-\varepsilon_{\mathrm{GS}}}{k_{\mathrm{B}} T}\right)+1},
$$

where $\varepsilon_{\mathrm{GS}}$ is the Fermi level and tends to the ground-state energy of the spin system in the limit of the temperature $T \rightarrow$ 0 , where $k_{\mathrm{B}}$ is the Boltzmann constant.

In the noninteracting case $\left(J_{s d}=0\right)$, the electronic system is not in equilibrium as the interaction with the electrodes establishes a steady state current. In contrast, the spin system is in thermal equilibrium at the temperature $T$. In this case, the energy resolved lesser and greater Green's functions take the form

$$
\begin{gathered}
{\left[G_{0}^{\lessgtr}(E)\right]_{\alpha \alpha^{\prime}}=\frac{\left[\Sigma_{\text {tip-S }}^{\lessgtr}(E)\right]_{\alpha \alpha^{\prime}}+\left[\Sigma_{\text {sub-S }}^{\lessgtr}(E)\right]_{\alpha \alpha^{\prime}}}{\left(E-\varepsilon_{0}\right)^{2}+\Gamma^{2}},} \\
{\left[D_{0}^{\lessgtr}(E)\right]_{m n}=\frac{\left[\Pi_{0}^{\lessgtr}(E)\right]_{m n}+\left[\Pi_{0}^{\lessgtr}(E)\right]_{m n}}{\left(E-\varepsilon_{m}\right)^{2}+\left(k_{\mathrm{B}} T\right)^{2}} .}
\end{gathered}
$$

In the electronic noninteracting Green's function [see Eq. (7)], the coupling of the sample to the tip and the substrate causes a broadening of the bare on-site level $\varepsilon_{0}$ of magnitude $\Gamma=\sum_{\eta \alpha \alpha^{\prime}}\left[\Gamma_{\eta-S}\right]_{\alpha \alpha^{\prime}}$, where $\eta=$ $\{$ tip,sub\}. The noninteracting self-energies take the form $\left[\Sigma_{\eta}^{>}(E)\right]_{\alpha \alpha^{\prime}}=\left[1-f_{\eta}(E, V)\right]\left[\Gamma_{\eta-S}\right]_{\alpha \alpha^{\prime}}$ and $\left[\Sigma_{\eta}^{<}(E)\right]_{\alpha \alpha^{\prime}}=$ $f_{\eta}(E, V)\left[\Gamma_{\eta-S}\right]_{\alpha \alpha^{\prime}}$, where $f_{\eta}(E, V)$ is the Fermi function in each of the $\eta$ th leads at a bias $V$.

In contrast, the local spin Green's function of Eq. (8) describes a system, which is adiabatically coupled to a heatbath of temperature $T$. This provides a very weak broadening of the single spin states $\varepsilon_{m}$ of magnitude $k_{\mathrm{B}} T$. Such a heat bath keeps the spin system in equilibrium and in the noninteracting case the population then resides mostly in the ground state [see Eq. (6)]. For a ground-state population of the spin system $P_{m}^{0}$, we have $\left[\Pi_{0}^{>}(E)\right]_{m n}=\delta_{m n}\left(1-P_{m}^{0}\right) k_{\mathrm{B}} T$ and $\left[\Pi_{0}^{<}(E)\right]_{m n}=\delta_{m n} P_{m}^{0} k_{\mathrm{B}} T$.

\section{B. Spin-polarized electron self-energy}

In order to evaluate the effects that the interaction has on the electronic motion, we must calculate the electronspin self-energy. Here, we take a perturbative approach and formally expand Eq. (3) up to the $n$th order in the interaction Hamiltonian $H_{e-s p}$ as

$$
\begin{aligned}
{\left[G\left(\tau, \tau^{\prime}\right)\right]_{\alpha \alpha^{\prime}}=} & \sum_{n} \frac{(-i)^{n+1}}{n !} \int_{C} d \tau_{1} \cdots \int_{C} d \tau_{n} \\
& \times \frac{\left\langle 0\left|T_{C}\left\{H_{e-s p}\left(\tau_{1}\right) \cdots H_{e-s p}\left(\tau_{n}\right) c_{\alpha}(\tau) c_{\alpha^{\prime}}^{\dagger}\left(\tau^{\prime}\right)\right\}\right| 0\right\rangle}{U(-\infty,-\infty)},
\end{aligned}
$$

where $U$ is the time-evolution unitary operator and the time averages are performed over the known noninteracting $\left(J_{s d}=\right.$ $0)$ ground state $|0\rangle$. The time integration over $\tau$ is ordered on the contour $C$ going from $-\infty$ to $+\infty$ and then returning from $+\infty$ to $-\infty$, since the ground state of the nonequilibrium system can only be defined at $-\infty$. $^{38}$
In the following, we consider the tip to have a spin polarization $\Lambda(-1<\Lambda<1)$. This is defined as the spin asymmetry in the electronic coupling between the tip and the sample. The spin-resolved electronic broadening is given by $\left[\Gamma_{\text {tip }-\mathrm{S}}\right]_{\uparrow \uparrow}=\frac{(1+\Lambda)}{2} \Gamma_{\text {tip-S }}$ and $\left[\Gamma_{\text {tip }-\mathrm{S}}\right]_{\downarrow \downarrow}=\frac{(1-\Lambda)}{2} \Gamma_{\text {tip-S }}$, where $\Gamma_{\text {tip-S }}$ is the non-spin-polarized broadening. ${ }^{4}$ The substrate is assumed to remain nonmagnetic. As a result, $\left[G_{0}^{\lessgtr}(E)\right]_{\uparrow \uparrow} \neq$ $\left[G_{0}^{\lessgtr}(E)\right]_{\downarrow \downarrow}$ so that we now must retain the spin indexes when constructing the self-energy. By using the same procedure described in Ref. 16, we can write the self-energy for the majority $(\uparrow)$ and minority $(\downarrow)$ spins, respectively, as

$$
\begin{aligned}
{\left[\Sigma_{\text {int }}^{\lessgtr}\right.} & (E)]_{\uparrow \uparrow}^{(2)} \\
= & -J_{s d}^{2} \sum_{m n}\left[G_{0}^{\lessgtr}\left(E \pm \Omega_{m n}\right)\right]_{\uparrow \uparrow} \\
& \times\left[\delta_{n m} \chi P_{n} S_{m n}^{z}+P_{n}\left(1-P_{m}\right)\left|S_{m n}^{z}\right|^{2}\right] \\
& -J_{s d}^{2} \sum_{m n}\left[G_{0}^{\lessgtr}\left(E \pm \Omega_{m n}\right)\right]_{\downarrow \downarrow} P_{n}\left(1-P_{m}\right)\left|S_{m n}^{ \pm}\right|^{2}
\end{aligned}
$$

and

$$
\begin{aligned}
{\left[\Sigma_{\text {int }}^{\lessgtr}\right.} & (E)]_{\downarrow \downarrow}^{(2)} \\
= & -J_{s d}^{2} \sum_{m n}\left[G_{0}^{\lessgtr}\left(E \pm \Omega_{m n}\right)\right]_{\downarrow \downarrow} \\
& \times\left[-\delta_{n m} \chi P_{n} S_{m n}^{z}+P_{n}\left(1-P_{m}\right)\left|S_{m n}^{z}\right|^{2}\right] \\
& -J_{s d}^{2} \sum_{m n}\left[G_{0}^{\lessgtr}\left(E \pm \Omega_{m n}\right)\right]_{\uparrow \uparrow} P_{n}\left(1-P_{m}\right)\left|S_{m n}^{\mp}\right|^{2} .
\end{aligned}
$$

The lesser (greater) self-energy describe an incoming (outgoing) electron that excites (relaxes) the spin system by $\Omega_{m n}=$ $\varepsilon_{m}-\varepsilon_{n}$, with a probability that depends on the occupation of the spin levels $P_{m}$ and $P_{n}$ and on the spin selection rules $S_{m n}^{z,+,-}$ (note $S^{+}=S^{x}+i S^{y}$ and $S^{-}=S^{x}-i S^{y}$ ). The first term in both Eqs. (10) and (11), proportional to $\delta_{n m}$, corresponds to the magnetoresistive elastic term of the $s$ - $d$ Hamiltonian (2). This represents an elastic contribution to the current since it does not involve a spin-flip transition, i.e., $\Omega_{m m}=0$. It is magnetic in nature since it involves the matrix element $S_{m m}^{z}$ and resistive since it affects to the current when the tip is polarized. The remaining contributions are inelastic and depend on the spin orientation of the electron transferred from the tip.

The action of the self-energy derived above is schematically explained in the diagram presented in Fig. 1, in which a tip probes a single atom deposited on a substrate. Let us assume that the atom is in its ground state, where the spin is in the up direction. If the tip is non-spin-polarized, the situation is that of Fig. 1(a), where inelastic steps in the conductance can be detected for both positive and negative biases. However, if the tip is spin polarized with spin-up polarization, electrons flowing at a positive bias (traveling from the tip to the substrate) can interact only elastically with the atom spin and therefore can tunnel out unperturbed through the substrate [see Fig. 1(b), positive bias]. On the contrary, when the bias is negative (electrons moving from the substrate to the tip), both spins are available for transport. Up-spin electrons may propagate only elastically, while down-spin electrons may 
(a)
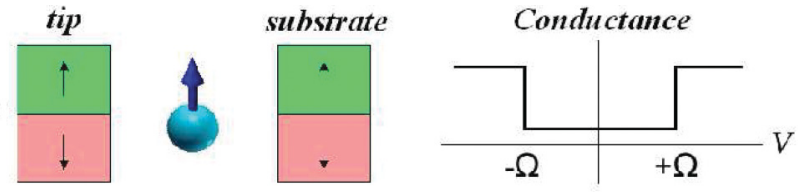

(b)
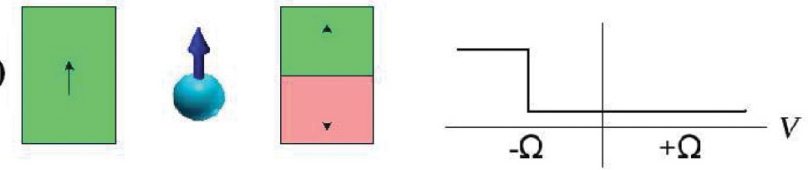

(c)
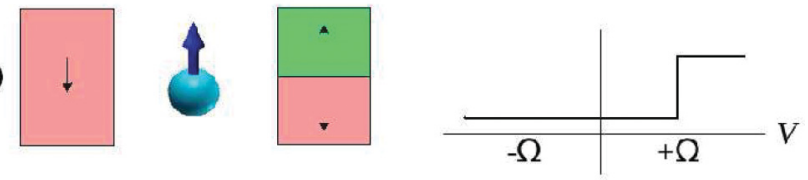

FIG. 1. (Color online) Cartoon describing the transport processes, where an atom deposited on a substrate is probed by a STM tip. Here, we assume that the atom is in its ground state with spin-up orientation. In (a), the tip is non-spin-polarized and inelastic steps in the conductance appear for both bias polarities. In (b) and (c), the tip is fully spin polarized with polarization respectively up (b) or down (c). This leads to a conductance step only for one of the two bias polarities.

undergo an inelastic interaction with the local spin and tunnel out through the tip as an up-spin electron. This process yields to the corresponding step in the conductance [see Fig. 1(b), negative bias]. The opposite scenario occurs when the tip is polarized along the down-spin direction [see Fig. 1(c)].

\section{Spin-polarized spin-self-energy}

When the atom is magnetic and the tunneling current tip carries a finite spin polarization the spin system can be dragged out of equilibrium, in particular, if the current density is intense. This means that the equilibrium conditions employed previously, ${ }^{16,17}$ namely, $P_{m=\mathrm{GS}}=1$ and $P_{m \neq \mathrm{GS}}=0$ are no longer valid. As a consequence, we must now derive also an expression for the propagator and thus for the selfenergy associated to the local spins. The derivation, up to second order in the electron-spin interaction, is described in detail in Appendix. In particular, the total spin self-energy also includes a zeroth-order contribution, which accounts for the noninteracting $\left(J_{s d}=0\right)$ case. This is approximated by $\left[\Pi_{0}^{<}(\omega)\right]_{k k}=P_{k}^{0} k_{\mathrm{B}} T$ and $\left[\Pi_{0}^{>}(\omega)\right]_{k k}=\left(1-P_{k}^{0}\right) k_{\mathrm{B}} T$, where $P_{k}^{0}$ is the ground-state population. Therefore, in absence of inelastic scattering, the spin system will remain in thermal equilibrium with the heat bath and only the ground state will be occupied.

By combining the zeroth- and second-order contributions to self-energy, we can write down a master equation, describing the nonequilibrium spin population, in terms of the total selfenergy $\Pi^{\lessgtr}(E){ }^{39}$

$$
\begin{aligned}
\frac{d P_{n}}{d t}= & \frac{1}{\hbar} \sum_{m} \int_{-\infty}^{+\infty} d E\left\{\left[\Pi^{>}(E)\right]_{n m}\left[D_{0}^{<}(E)\right]_{m n}\right. \\
& \left.\left.-\Pi^{<}(E)\right]_{n m}\left[D_{0}^{>}(E)\right]_{m n}\right\} .
\end{aligned}
$$

After some rearrangement, this can be written in more compact form as

$$
\begin{aligned}
\frac{d P_{n}}{d t}= & \sum_{l}\left[P_{n}\left(1-P_{l}\right) W_{l n}-P_{l}\left(1-P_{n}\right) W_{n l}\right] \\
& +\left(P_{n}^{0}-P_{n}\right) k_{\mathrm{B}} T,
\end{aligned}
$$

where the bias-dependent transition rate from an initial state $|l\rangle$ to a final state $|n\rangle$ is calculated after evaluating the integral described in Eq. (A7). This finally writes

$$
\begin{aligned}
W_{n l}= & -4 \frac{\left(\rho J_{s d}\right)^{2}}{\Gamma} \sum_{\eta \eta^{\prime}} \zeta\left(\mu_{\eta}-\mu_{\eta^{\prime}}+\Omega_{l n}\right) \\
& \times\left\{\chi S_{n n}^{z}\left(\left[\Gamma_{\eta}\right]_{\uparrow \uparrow}\left[\Gamma_{\eta^{\prime}}\right]_{\uparrow \uparrow}-\left[\Gamma_{\eta}\right]_{\downarrow \downarrow}\left[\Gamma_{\eta^{\prime}}\right]_{\downarrow \downarrow}\right)\right. \\
& +\left|S_{n l}^{z}\right|^{2}\left[\Gamma_{\eta}\right]_{\uparrow \uparrow}\left[\Gamma_{\eta^{\prime}}\right]_{\uparrow \uparrow}+\left|S_{n l}^{z}\right|^{2}\left[\Gamma_{\eta}\right]_{\downarrow \downarrow}\left[\Gamma_{\eta^{\prime}}\right]_{\downarrow \downarrow} \\
& \left.+\left|S_{n l}^{+}\right|^{2}\left[\Gamma_{\eta}\right]_{\downarrow \downarrow}\left[\Gamma_{\eta^{\prime}}\right]_{\uparrow \uparrow}+\left|S_{n l}^{-}\right|^{2}\left[\Gamma_{\eta}\right]_{\uparrow \uparrow}\left[\Gamma_{\eta^{\prime}}\right]_{\downarrow \downarrow}\right\},
\end{aligned}
$$

where $\zeta(x)=x /\left(1-e^{-x / k_{\mathrm{B}} T}\right)$ and $\mu_{\eta}$ is the chemical potential in lead $\eta=\{$ tip, sub $\}$. Note that $\zeta(x)$ is such that for $\eta=\eta^{\prime}$ the resulting transition rates $W_{n l}$ are bias independent and do not contribute to the current. However, they do contribute to the spin relaxation time, i.e., to the time taken by the localized spin system to relax back to its equilibrium state. Such a relaxation time is reduced if the coupling between the sample and the leads is increased. Furthermore, the smaller is the inelastic energy transition $\Omega_{m n}$, the longer the spins will remain in the excited state before relaxing back to equilibrium. Finally, we note that the above expression is based on the assumption that the on-site energy is large enough for the density of states of the spin system to remain constant in the small energy window of interest. Therefore $\rho=\Gamma /\left(\varepsilon_{0}^{2}+\Gamma^{2}\right)$.

Returning to Eq. (13) note that we are only interested in the steady state nonequilibrium population of the spin states at a given bias. Therefore we can set $d P_{n}(t) / d t=0$ and reduce Eq. (13) to a system of linear equations, which can be solved self-consistently. For an initial guess of the populations $\left(P_{l}=P_{l}^{0}\right)$, we can iterate Eq. (13), which define $P_{n}$, with Eq. (A7), which define the self-energy $\Pi \lessgtr$, until self-consistency is reached. We can then combine the resulting nonequilibrium population with the second-order electronic self-energy calculated in Eq. (3) to obtain the current.

\section{Real part of the electron self-energy}

In order to provide an explanation to the inherent bias asymmetry that has been observed in most of the STM inelastic conductance spectra for magnetic atoms probed by a nonmagnetic STM tip, we return to the expression for the full retarded self-energy. This is defined by the Hilbert transform (note we will only consider the non-spin-polarized case for simplicity):

$$
\begin{aligned}
\Sigma_{\text {int }}(E)= & \mathcal{P} \mathcal{V} \int_{-\infty}^{+\infty} \frac{d E^{\prime}}{2 \pi} \frac{\Sigma_{\text {int }}^{>}\left(E^{\prime}\right)+\Sigma_{\text {int }}^{<}\left(E^{\prime}\right)}{E-E^{\prime}} \\
& +\frac{i}{2}\left\{\Sigma_{\text {int }}^{>}\left(E^{\prime}\right)+\Sigma_{\text {int }}^{<}\left(E^{\prime}\right)\right\} .
\end{aligned}
$$

By using the expressions derived in Sec. II C for the secondorder lesser and greater self-energies, we can find an analytic 
expression for the real contribution to the retarded self-energy:

$$
\begin{aligned}
\operatorname{Re}[ & \left.\Sigma_{\text {int }}(E)^{(2)}\right] \\
= & 2 \rho J_{s d}^{2} \sum_{i, m, n}\left|S_{m n}^{i}\right|^{2} P_{n}\left(1-P_{m}\right) \times \frac{1}{\Gamma}\left\{2 \pi \varepsilon_{0}+\sum_{\eta} \Gamma_{\eta-\mathrm{S}}\right. \\
& \left.\times \ln \left[\frac{\left(E+\Omega_{m n}-\mu_{\eta}\right)^{2}+\left(k_{B} T\right)^{2}}{\left(E-\Omega_{m n}-\mu_{\eta}\right)^{2}+\left(k_{B} T\right)^{2}}\right]\right\}
\end{aligned}
$$

Such a final expression is heavily dependent on the on-site energy $\varepsilon_{0}$ but also note the ratio $\Gamma_{\text {tip-s }} / \Gamma_{\text {sub-s }}$ has a profound influence on the degree of asymmetry present in the conductance spectrum. If $\Gamma_{\text {tip-S }} / \Gamma_{\text {sub-S }}=1$ then the asymmetry is removed. Provided that $\Gamma_{\text {tip-S }}<\Gamma_{\text {sub-s }}$, which is a condition normally met in STM-IETS experiments, an asymmetry will always be present. This is a result also found in references. ${ }^{27,28}$ The condition $\Gamma_{\text {tip-S }}<\Gamma_{\text {sub-s }}$ makes the real part of the self-energy an odd function of the energy and the bias via its logarithmic dependence on the spin eigenvalues with opposite polarity for $+\Omega_{m n}$ and $-\Omega_{m n}$. Therefore one of the main physical causes of the bias asymmetry in the conductance spectra is the uneven electronic couplings between the scattering atoms and two noninteracting leads. As such, this is a feature common to all IETS experiments, including those using non-spin-polarized tips or scattering to phonon. In this work, we solely focus on non-spin-polarized tip and spin excitations.

\section{E. Electronic current}

We can finally unveil the effects that a nonequilibrium spin-population bares on the conductance profile of a magnetic nanostructure by taking the derivative of the current with respect to the bias voltage $V$. The current, $I_{\eta}$, flowing at the electrode $\eta=\{$ tip, sub $\}$ can be written as

$$
\begin{gathered}
I_{\eta}=\int_{-\infty}^{+\infty} d E \bar{I}_{\eta}(E), \\
\bar{I}_{\eta}(E)=\frac{q}{h} \operatorname{Tr}\left\{\left[\Sigma_{\eta}^{>}(E) G^{<}(E)\right]-\left[\Sigma_{\eta}^{<}(E) G^{>}(E)\right]\right\},
\end{gathered}
$$

where $G \lessgtr(E)$ are the full many-body lesser/greater electronic Green's functions. These are finally defined as

$$
\left[G^{\lessgtr}(E)\right]=\frac{\left[\Sigma_{\text {tip-S }}^{\lessgtr}(E)\right]+\left[\Sigma_{\text {sub-S }}^{\lessgtr}(E)\right]+\left[\Sigma_{\text {int }}^{\lessgtr}(E)\right]}{\left\{E-\varepsilon_{0}-\operatorname{Re}\left[\Sigma_{\text {int }}(E)\right]\right\}^{2}+\left\{\Gamma-\operatorname{Im}\left[\Sigma_{\text {int }}(E)\right]\right\}^{2}},
$$

and $\left[\Sigma_{\text {int }}^{\lessgtr}(E)\right]=\left[\Sigma_{\text {int }}^{\lessgtr}(E)\right]^{(2)}+\left[\Sigma_{\text {int }}^{\lessgtr}(E)\right]^{(3)}$, where the thirdorder self-energies are calculated following Ref. 17. Here, for simplicity, we take the expression for the third-order contribution to $\Sigma_{\text {int }}$ obtained by neglecting any explicit spin polarization. Such approximation is justified by the fact that the effects due to spin polarization are small at the third order and that in doing so we avoid a rather cumbersome formulation. We do, however, consider the combination of non-spin-polarized third-order effects with second-order spinpolarized self-energies to highlight subtle differences in the spectra.

\section{RESULTS}

\section{A. Nonequilibrium population}

We start our analysis by first looking at the effects originating from driving the spin system out of equilibrium with an electronic current. This attempts at explaining the experiments reported in Ref. 4 in which an STM tip (nonmagnetic) is positioned above a $\mathrm{Mn}$ dimer deposited onto a $\mathrm{CuN}$ substrate. The conductance spectra are measured for different tip to sample distances. Varying the STM tip height is equivalent to changing both the current density and the electronic coupling between the tip and the sample. Nonequilibrium effects then appear as variations of the conductance profiles as a function of the STM tip height. Many of the parameters needed by our model can be extrapolated from a similar experiment carried on over Mn linear atomic chains deposited on $\mathrm{CuN}^{3}$ The five unpaired electrons in the Mn $3 d$ shell suggest a $S=5 / 2$ ground state, as confirmed both by experiments and theory. ${ }^{14,24}$ The spin-spin exchange interaction between two $\mathrm{Mn}$ atoms is antiferromagnetic and has an estimated value of $J_{d d}=6.2 \mathrm{meV}$. As a result, the ground state of the dimer is a singlet (total spin $S=0$ ). The first excited state is a triplet with total spin $S=1$ and the energy splitting between the ground state and such first excited state is exactly $J_{d d}$. The next excited level is the quintuplet with total spin $S=2$ and it is separated from the first excited state by $2 J_{d d}$. This pattern continues throughout the spin manifold (see Fig. 2). The axial and transverse anisotropies are found to be $D=-0.037 \mathrm{meV}$ and $E=0.007 \mathrm{meV}$, respectively. These cause the lifting of the spin multiplets degeneracy. The temperature is set at $T=0.5 \mathrm{~K}$. The value of $J_{s d}$ is estimated from density functional theory (DFT) to be of the order of $500 \mathrm{meV},{ }^{40}$ while $\Gamma_{\text {sub-S }}$ is also found from DFT to be approximately $100 \mathrm{meV} .{ }^{19}$ In contrast, $\Gamma_{\text {tip-s }}$ remains an adjustable parameter with the chosen values ranging from 0.125 to $200 \mathrm{meV}$. Finally, in order to ensure a nearly constant density of states around the Fermi energy $\left(E_{F}=0\right)$, we set the on-site energy of the atom under the STM tip to be $\varepsilon_{0}=1 \mathrm{eV}$.

Figure 3 shows the conductance spectra (normalised by $G_{\text {el }}$, the conductance calculated when $J_{s d}=0$ ) obtained by simply taking the numerical derivative of the current [see Eq. (17)] with respect to the bias. We consider three different tip to sample distance, corresponding respectively to weak $\left(\Gamma_{\text {tip-S }}=0.5 \mathrm{meV}\right)$, intermediate $\left(\Gamma_{\text {tip-s }}=50 \mathrm{meV}\right)$ and strong $\left(\Gamma_{\text {tip-s }}=200 \mathrm{meV}\right)$ electronic coupling. The evolution of

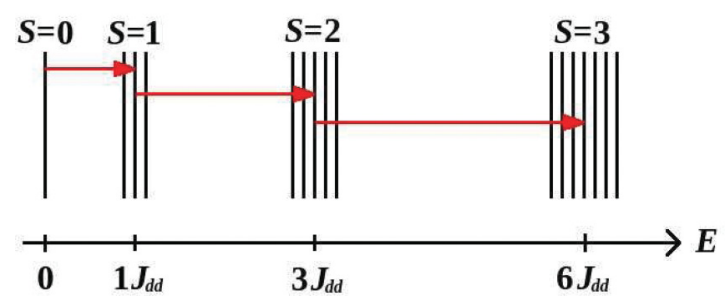

FIG. 2. (Color online) Excitation spectrum for an antiferromagnetically exchanged coupled $\mathrm{Mn}$ dimer deposited on $\mathrm{CuN}$. The ground state is a $S=0$ spin singlet. The first three excited state multiplets have respectively spin $S=1,2$, and 3. In the figure, we also indicate the energy separation between the various spin multiplets in units of the exchange parameter $J_{d d}$. 


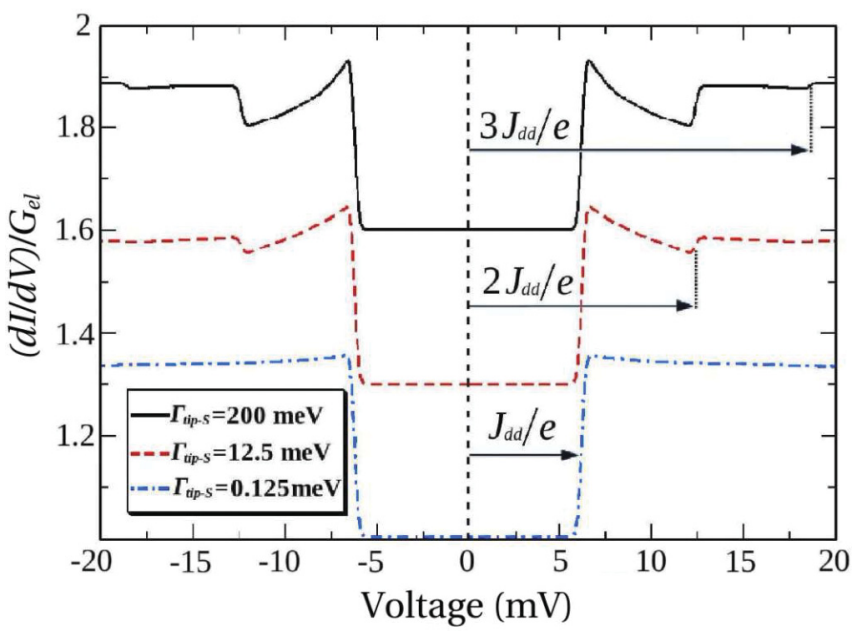

FIG. 3. (Color online) Normalized conductance spectra for the Mn dimer calculated at different tip to sample distance, i.e., for different $\Gamma_{\text {tip-s }}$ coupling strengths. We notice that the stronger is the coupling, the more the system is driven out of equilibrium. This results in the appearance of additional spin transitions, which manifest themselves as steps or drops of the conductance as a function of bias.

the conductance line shape as a function of $\Gamma_{\text {tip-S }}$ is a direct consequence of the spin system being driven out of equilibrium. For $\Gamma_{\text {tip-S }}=0.5 \mathrm{meV}$, the STM tip is far enough from the sample to ensure that the spin system is always in its ground state between two subsequent electron tunneling events. Therefore the only transition detected in the $G(V)$ profile is that between the $S=0$ ground state and the first excited state with $S=1$. This has an excitation energy equal to $J_{d d}$ and it does manifest itself as a conductance step at a voltage $V=J_{d d} / e$, with $e$ being the electron charge.

As the tip is brought closer to the sample $\left(\Gamma_{\text {tip-S }}=50 \mathrm{meV}\right)$, the first excited triplet level $(S=1)$ starts to populate. Now an incoming electron with sufficiently large energy $\left(2 J_{d d}\right)$ can induce a second transition from from the first to the second excited state. Note that a spin transition from the ground state to the $S=2$ state is highly unlikely with a single electron tunneling process. This would be completely suppressed in the case of no magnetic anisotropy and it is expected to have a negligible intensity for finite, but small, values. Note that such transition will become more probable if the spin system does not have enough time between tunneling events to relax back to the ground state. For this case, the transition appears as an increase of the conductance at the critical voltage $V=2 J_{d d} / e$ with a subsequent decay. The same spectroscopical feature is further enhanced at an even larger current density $\left(\Gamma_{\text {tip-S }}=\right.$ $200 \mathrm{meV}$ ), when a third conductance step appears at $3 J_{d d} / e$. This is associated to a transition from the $S=2$ to the $S=3$ spin state and it becomes possible only if the occupation of the $S=2$ level is not zero, i.e., if the system is driven to this highly excited state. It is clear that by appropriately fixing the two adjustable parameters $\varepsilon_{0}$ and $\Gamma_{\text {tip-s }}$ we can achieve almost perfect quantitative agreement with the experimental data (see Fig. 2 of Ref. 4). The evolution of the population of the various spin states (up to $S=2$ ) as a function of bias is presented in Fig. 4. This is calculated in the case of strong tip to sample electronic coupling $\Gamma_{\text {tip-S }}=200 \mathrm{meV}$. In the figure, one can

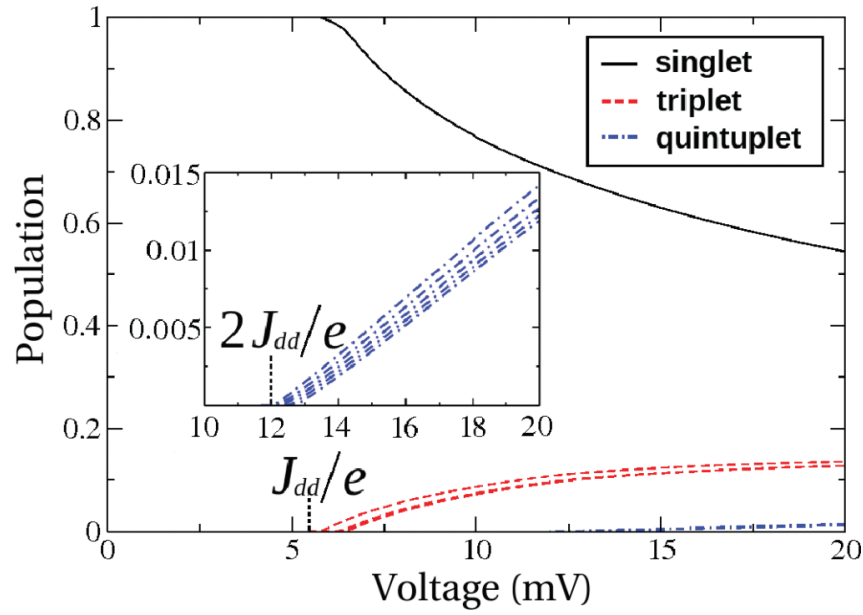

FIG. 4. (Color online) Nonequilibrium population of the Mn dimer singlet $(S=0)$, triplet $(S=1)$, and quintuplet $(S=2)$ states. The inset shows a magnified view of the population of the $S=2$ state as it start to get populated at approximately $12 \mathrm{mV}$.

note the strong spin pumping from the ground state into both the first and the second excited state. The excitation to the third excited state occurs at approximately $18 \mathrm{meV}$ but is too weak to be observed on this scale.

The reason for the enhancement of the conductance step and the subsequent decay can be attributed to the bias dependence of the spin population, as described in Fig. 4. We note that there is a large dip in the ground-state population at the point when the first transition occurs. Since we eventually wish to calculate the conductance, we must evaluate the derivative of the population with respect to the bias, which, to a good approximation, will have the appearance of a Lorentzian peak. This ultimately contributes to the conductance at the inelastic step as a peak and a subsequent dip (see Fig. 3). Such an effect will only appear when the system is driven out of equilibrium by increasing the tip-sample coupling $\Gamma_{\text {tip-s }}$. However, a second effect can also generate a rather similar line shape, namely, the inclusion in the perturbative expansion of third-order contributions, presenting a Kondo-like logarithmic divergence, in the self-energy.

In order to make this point more clear and to qualitatively distinguish between these two possible sources of line shape, we look in some details at the case of a single $\mathrm{Mn}$ atoms probed by a non-spin-polarized tip, whose conductance spectrum is presented in Fig. 5. Clearly, we observe that increasing $\Gamma_{\text {tip-S }}$ has minimal effect on the conductance line shape. Even when $\Gamma_{\text {tip-s }}=200 \mathrm{meV}$ and the population is driven far from equilibrium, as is seen in Fig. 6, we detect no significant conductance overshoot at the bias step. This effect has been explored by Novaes et al. in Ref. 24, where it is claimed that the conductance overshoot brought about by inelastic transitions is balanced by elastic transitions that do not alter the state of the local spin, resulting in a flat spectrum. This is in conflict with what is found in experiments, where a visible conductance peak and subsequent decay is observed at the bias step. ${ }^{4}$ Therefore we claim that third order effects, where the logarithmic peak in this case is dependent on the parameter $\rho J_{s d}$ and therefore exists even at equilibrium, ${ }^{17}$ are the main 


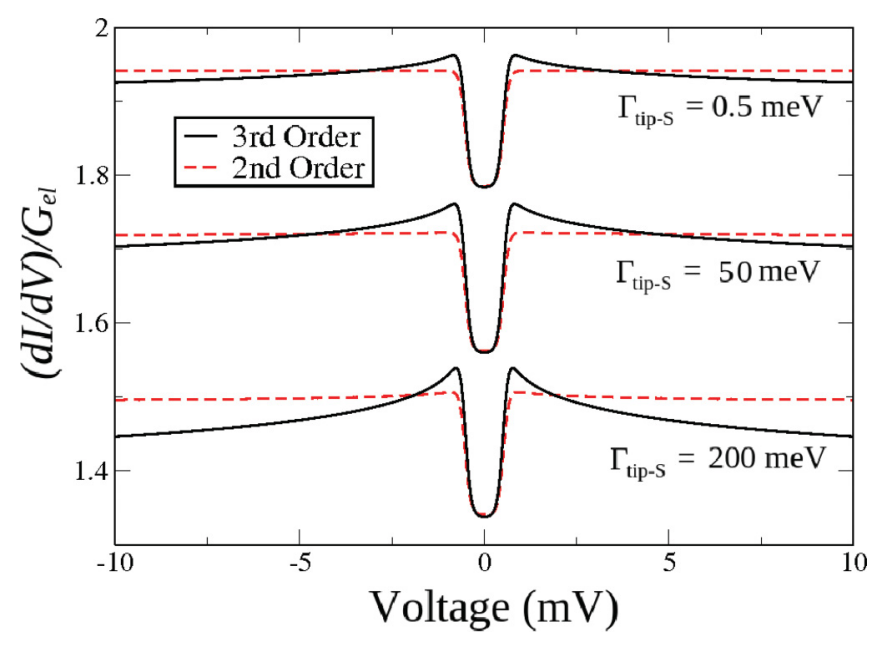

FIG. 5. (Color online) Normalized conductance spectra for a single $\mathrm{Mn}$ atom explored by a non spin-polarized STM tip at different tip to sample electronic couplings $\Gamma_{\text {tip-s }}$. Here, a magnetic field of $3 \mathrm{~T}$ is applied along the $z$ axis. Comparison is made between second- and third-order calculations.

contributors to the observed nonlinear conductance. This can be easily seen from the third-order contribution to Fig. 5 .

\section{B. Spin-polarized tip}

We now move on to consider the situation where the tip is magnetic, i.e., when the injected current is spin polarized. Again, we use as guide the experimental work of Loth et al. ${ }^{4,9}$ The STM tip is now spin polarized by placing an additional Mn atom at its apex, while also applying a strong magnetic field perpendicular to the substrate of $3 \mathrm{~T}$. In this case, the spectrum is collected from a single $\mathrm{Mn}$ or $\mathrm{Fe}$ ion on the surface (not from a dimer). In the case of $\mathrm{Mn}$, the atom exhibits a weak anisotropy on $\mathrm{CuN}$ (see previous section) and the strong magnetic field effectively produces a Zeeman split of the six levels of the $S=5 / 2 \mathrm{Mn}$ spin manifold. The direction of the magnetic field in these experiments is

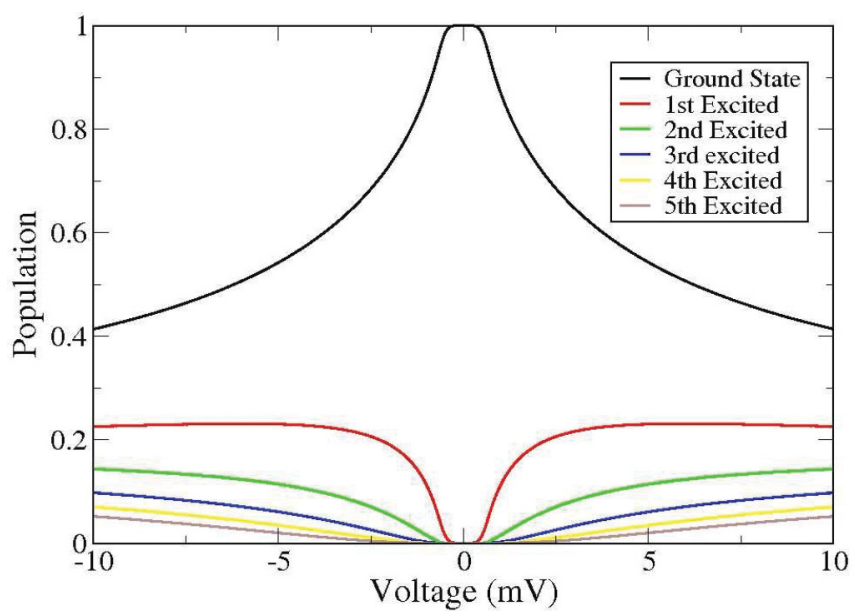

FIG. 6. (Color online) Nonequilibrium population of the various spin states of $\mathrm{Mn}$ on $\mathrm{CuN}$ as a function of bias for non-spin-polarized tip.

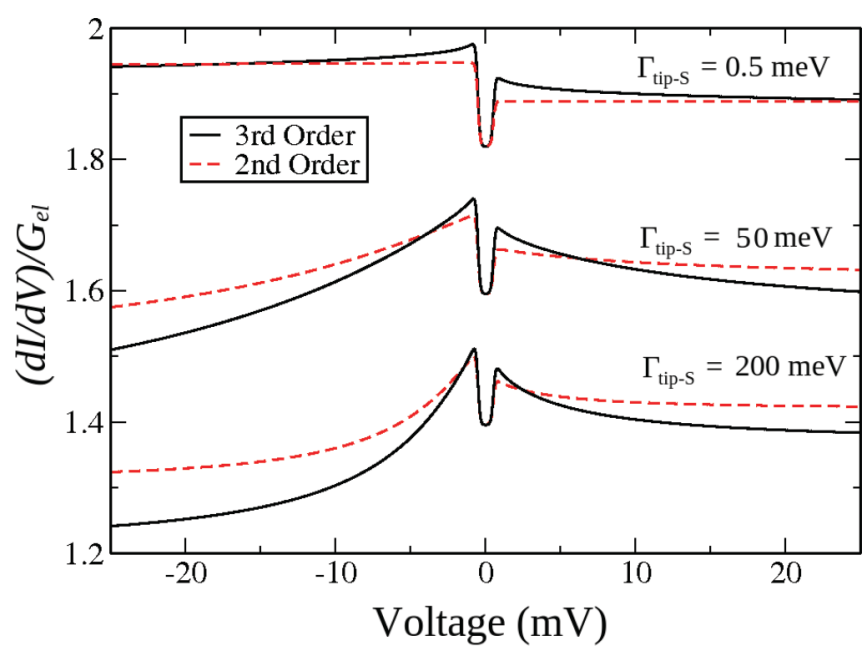

FIG. 7. (Color online) Normalized conductance spectra for a single $\mathrm{Mn}$ atom explored by spin-polarized STM tip at different tip to sample electronic couplings $\Gamma_{\text {tip-s }}$. Here, a magnetic field of $3 \mathrm{~T}$ is applied along the $z$ axis. The asymmetry in the conductance profile is is due to the spin-polarization of the tip. Such an asymmetry is more pronounced as the system is driven further away from equilibrium. Comparison is made between second- and third-order calculations.

chosen so that the ground state of the Mn spin corresponds to the magnetic quantum number $m=+5 / 2$. Since the same magnetic field is applied to the Mn atom on the tip's apex, the tip and atom are both spin polarized and collinear. Figure 7 shows the calculated spectra for the system described above. In particular, we consider magnetic field strength of $3 \mathrm{~T}$ and either weak $\left(\Gamma_{\text {tip-S }}=0.5 \mathrm{meV}\right)$, intermediate $\left(\Gamma_{\text {tip-s }}=50 \mathrm{meV}\right)$, or strong $\left(\Gamma_{\text {tip-S }}=200 \mathrm{meV}\right)$ tip to sample couplings. The on-site energy is fixed at $\varepsilon_{0}=1.5 \mathrm{eV}$ and the value of $J_{s d}=1 \mathrm{eV}$ is inferred from the work of Lucignano et al., ${ }^{40}$ which results in an inelastic ratio of $\chi=1.5$. The tip spin-polarization constant that best fits the experimental data is $\eta=-0.3$. In the weak coupling regime (when the local spin remains always close to equilibrium), the local spin resides almost entirely in its $m=+5 / 2$ ground state. Due to the spin-exchange selection rules and to the collinearity of the tip and the sample, only the minority carriers can excite the local spin out of the ground state. For a tip spin polarization of $\Lambda=-0.3$, there are more minority electrons coming from the tip than those coming from the substrate. As a result, the intensity of the inelastic interaction will change depending on the direction of the current. This creates an asymmetry in the conductance spectrum with respect to the applied bias. The additional line shape features appearing in the weak coupling case (the conductance decay following a conductance step) are due to the third order Kondo-like self-energy, which produces a logarithmic decay at the conductance steps. This result is in good agreement with experiments (see Fig. 4 of Ref. 4).

When the spin of the $\mathrm{Mn}$ ion is driven further out of equilibrium, in particular in the strong coupling case, the bias asymmetry becomes more pronounced. Such spin-pumping phenomenon can be appreciated by looking at Fig. 8(a), where we show the populations of the six spin states of the $\mathrm{Mn}$ atom as a function of bias for strong tip to sample coupling $\left(\Gamma_{\text {tip-S }}=200 \mathrm{meV}\right)$. From the figure, one can see 

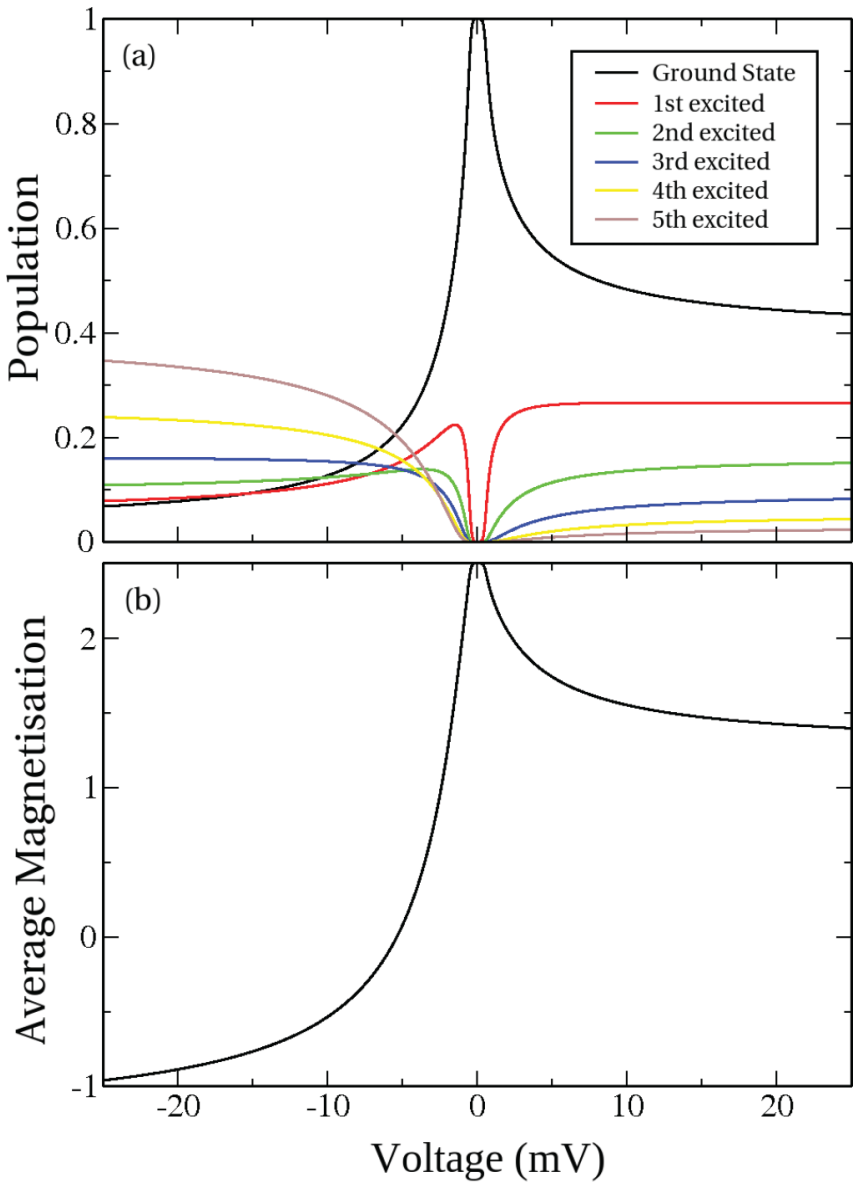

FIG. 8. (Color online) (a) Nonequilibrium population of the various spin states of $\mathrm{Mn}$ on $\mathrm{CuN}$ as a function of bias for spinpolarized tip and (b) the resulting average magnetization. These have been calculated for a magnetic field of $7 \mathrm{~T}$ aligned along the $z$ axis and for strong tip to sample electronic coupling $\Gamma_{\text {tip-s }}=200 \mathrm{meV}$. We notice that as the spin is driven far away from its equilibrium ground state the magnetization flips its direction.

that as the bias increases the $m=+5 / 2$ ground state gets depleted in favor of populating the other five excited states. In particular, already at around $V=-10 \mathrm{mV}$, the population of the $m=-5 / 2$ level is larger than that of the ground state. In the figure, we also plot the average magnetization, which is defined as $\left\langle S^{z}\right\rangle=\sum_{m} P_{m} S_{m m}^{z}$ [see Fig. 8(b)]. Intriguingly, we find that for negative biases, the spin is effectively flipped from $m=+5 / 2$ to $m=-1$ over a $25 \mathrm{meV}$ range. In this case, we encounter a similar situation to that presented in Fig. 1(c) where the local spin is no longer collinear to the tip polarization, and hence it results in a weaker inelastic signal for negative bias than that observed for positive. Therefore we observe a large dip in the conductance at negative biases when the spin is driven far from equilibrium and its polarization is effectively switched.

In Fig 9, we present the calculated spectra for the Fe atom in the spin-polarized case. We choose parameters in this case that conform with the experimental data of Loth et al. ${ }^{9}$ The Fe atom is assumed to carry a quantum mechanical spin of $S=2$ and it also exhibits a transverse easy axis anisotopy of $D=-1.53 \mathrm{meV}$ and an axial anisotropy of $E=0.31 \mathrm{meV}$.
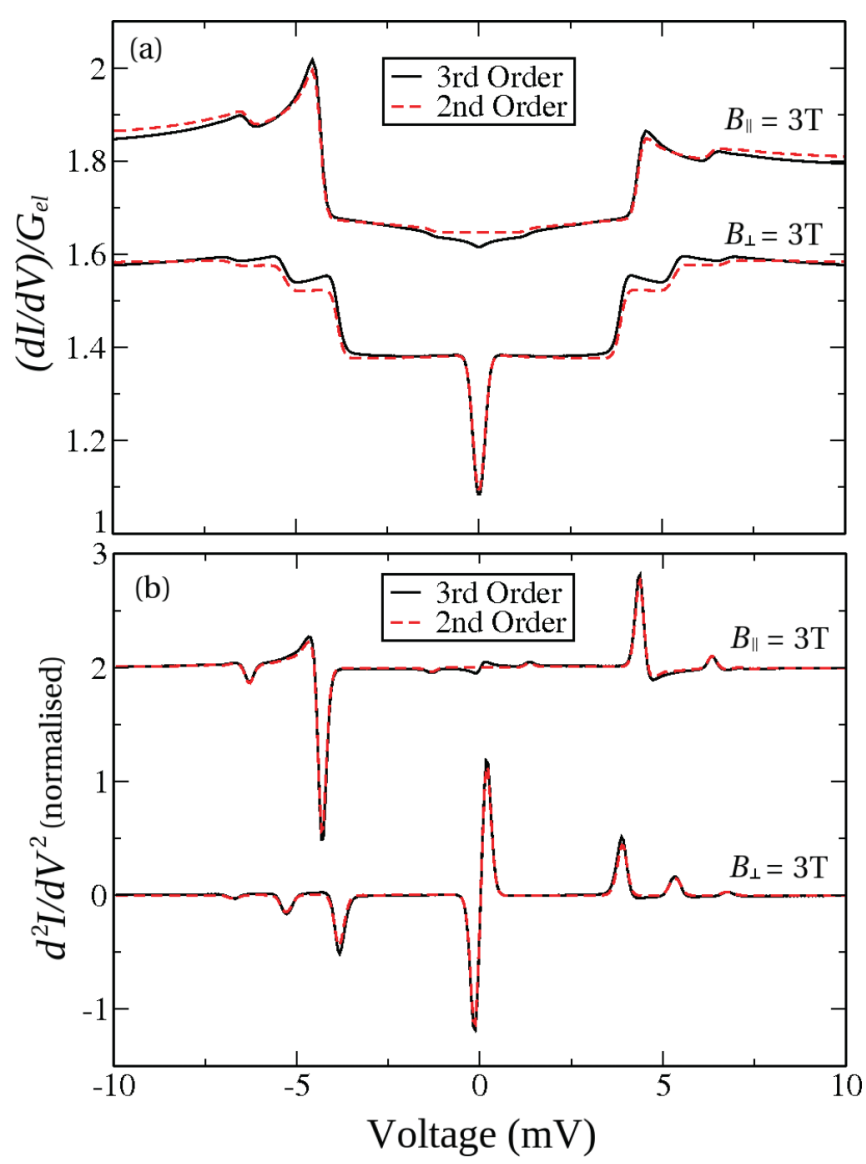

FIG. 9. (Color online) (a) Second- and third-order conductance spectra for the Fe atom with spin polarization $\eta=0.35$. The magnetic field of $3 \mathrm{~T}$ is applied both parallel and perpendicular to the easy axis of the Fe atom. (b) Second derivative of the current for the same spectra shown in (a).

We again assume a large value of the onsite energy, $2 \mathrm{eV}$, and we examine the spectra in the strong coupling case of $\Gamma_{\text {tip-s }}=$ $200 \mathrm{meV}$ with a tip polarization of $\eta=0.35$ and magnetic field strength $3 \mathrm{~T}$ as used in experiments. In Fig. 9(a), we present the conductance spectra for the two cases when the magnetic field is parallel and perpendicular to the easy axis of the atom (the $z$ axis in this model). As previously, we present this for both second- and third-order calculations. Firstly, we notice that the spin-polarized tip affects the spectra only in the case of parallel magnetic field where a clear bias assymetry is produced. No significant assymetry is found in the perpendicular case. This conforms with the experimental findings and is due to the fact that electron spins in the tip are no longer colinear with localized spin of the Fe atom.

As found in previous works, ${ }^{17}$ the inclusion of third-order effects is vital in reproducing the correct logarithmic decay at each of the conductance steps, which is particularly noticable for the perpendicular magnetic field. More significantly, experimental spectra for the parallel case exhibit a zero-bias conductance dip, which is absent in the second-order spectra but appears strongly when third-order effects are included. This can also be seen from the calculation of the second derivative of the current in Fig. 9(b) where a clear zero bias anomaly is evident in the third-order case. 


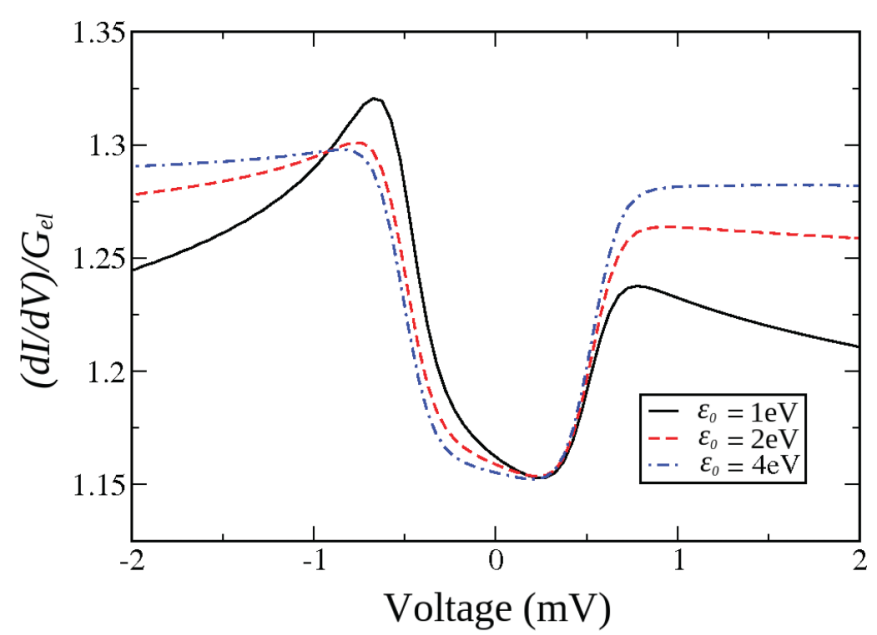

FIG. 10. (Color online) Normalized conductance spectra for a single Mn single calculated by including the real contribution to the interacting electron-spin self-energy for different on-site energies $\varepsilon_{0}$. Note that the conductance asymmetry increases with decreasing $\varepsilon_{0}$, i.e., as the onsite energy moves closer to the Fermi level.

\section{Non-spin-polarized asymmetry}

We finally move to discuss the inherent asymmetry measured in the conductance profile, which is usually observed even if the tip is not spin polarized. ${ }^{4,7}$ We model this line-shape feature by including the real part of the full interacting electron-spin self-energy in the description [see Eq. (16)]. The structure of this contribution to the self-energy shows an explicit dependence on the on-site energy, $\varepsilon_{0}$, and also a logarithmic peak of width $k_{\mathrm{B}} T$ at the onset of an inelastic transition $\left(E-\mu_{\eta}= \pm \Omega_{m n}\right)$. The asymmetry arises from the difference in polarity of the logarithmic peak for $\pm \Omega_{m n}$. The self-energy is thus an odd function of both energy and bias. This results in the conductance profile having a decrease of the step heights for $V=-\Omega_{m n} / e$ and a increase of them for $V=+\Omega_{m n} / e$.

We test this approach by considering the case of a nonspin-polarized tip and a single $\mathrm{Mn}$ atom. We use the same anisotropy parameters as for the Mn dimer but, for the sale of simplicity, we keep the spin always its equilibrium state and choose $\Gamma_{\text {tip-s }}=0.5 \mathrm{meV}$. Figure 10 shows the resulting conductance spectra for three different choices of the on-site energy $\varepsilon$. It is clear that the closer $\varepsilon$ is to the Fermi energy $(0 \mathrm{eV})$, the greater is the bias asymmetry, while as $\varepsilon$ is increased, the conductance profile becomes more symmetric. In this respect, the formalism outlined here is in agreement with the Fano line-shape argument ${ }^{41}$ where the degree of asymmetry for electrons tunneling through a single impurity is given by a ratio of the real to the imaginary contributions to the interacting Green's function. ${ }^{42}$

As an additional test, we consider the case of a $\mathrm{Mn}$ trimer, whose spectrum was shown first by Hijibehedin et al. ${ }^{3}$ to exhibit a large bias asymmetry when measured with a nonmagnetic tip. We model this system by choosing an antiferromagnetic nearest neighbor exchange coupling $J_{d d}^{(1)}=$ $2.3 \mathrm{meV}$. Furthermore, in order to accurately describe the position of the principle conductance steps in the conductance

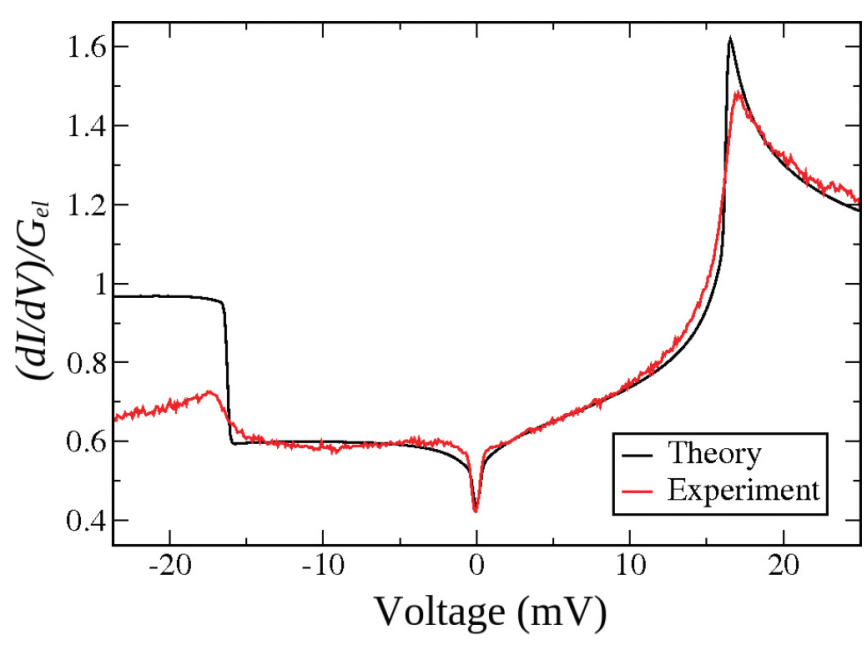

FIG. 11. (Color online) Comparison between the experimental (red) and theoretical (black) conductance spectra for a Mn trimer on $\mathrm{CuN}$ probed by a nonmagnetic STM tip. In the calculation, we have included the real part of the interacting electron-spin self-energy. This provides the conductance asymmetry with bias.

profile, we also include a ferromagnetic second-nearestneighbour interaction between the local spins of magnitude $J_{d d}^{(2)}=-1.0 \mathrm{meV}^{13}$

We again choose to keep the spin system in equilibrium and therefore consider weak coupling between the STM tip and the second atom in the trimer chain $\left(\Gamma_{\text {tip-s }}=0.5 \mathrm{meV}\right)$. The best fit to the experimental data is found with $\varepsilon_{0}=-1 \mathrm{eV}$. Figure 11 shows the model fit to the experimental data (from Ref. 3). Whereas previous calculations did not predict any conductance asymmetry, ${ }^{16}$ it is clear from the figure that the inclusion of the real part of the self-energy in the description produces a significant conductance asymmetry. This is most prominent at the principle step height $(\approx \pm 16.5 \mathrm{meV})$ for each bias polarity. Although the step height for the negative bias is not as small as that found experimentally, the qualitative trends are similar. In particular, we notice the logarithmic conductance increase (reduction) that occurs before (after) the onset of the step at $V=+16.5 \mathrm{meV}$, which also originates from the third-order contribution to the self-energy.

In this work, based on a perturbative approach of the $s$ - $d$ model, we have shown that the entire line-shape description can be reconciliated with experiments by considering an expansion of the self-energy to the third order, which also includes its real part. As such, we have shown that the conductance asymmetry can be described also if the electronic orbitals forming the samples spin are not explicitly taken into account as also suggested by Delgado and FernandezRossier. $^{23}$

\section{CONCLUSIONS}

In conclusion, we have studied the line-shape details of the conductance profile of $\mathrm{Mn}$ atoms deposited on $\mathrm{CuN}$ and probed with a STM tip, which can or can not carry spin-polarization. In particular, we have looked closely at the asymmetry of the conductance with the bias polarity. Firstly, 
we have extended our perturbative approach to spin scattering to the spin-polarized case and considered an expansion of the complex part of the electronic propagator up to the third order. This allows us to reproduce the logarithmic decay of the conductance subsequent to a conductance step, which is observed in experiments but could not be explained by a second-order theory.

When the current density is increased and the tip is spin polarized, the conductance profile starts to develop a significant asymmetry with respect to the bias polarity. These are indicative of the spin system being driven out of equilibrium. We have then derived a second-order expansion of the spin propagator capable of evaluating the nonequilibrium population of the various spin energy levels. This was put favorably to the test against a series of experiments probing a single $\mathrm{Mn}$ and Fe ions with a spin-polarized STM tip in an intense magnetic field. Furthermore, the same formalism was capable of describing excitations occurring away from the ground state for a Mn dimer probed by a nonmagnetic tip. Also in this case, the agreement with experiments is very satisfactory.

Finally, in an attempt to describe the bias asymmetry in the case of non-spin-polarized STM tips, we have derived an analytic expression for the real part of the electron-spin interacting self-energy. This contains logarithmic peaks at the excitation energies that are odd with respect to energy and voltage. Such parity results in an asymmetry in the conductance profiles. Such a scheme was tested for the case of a $\mathrm{Mn}$ monomer and a $\mathrm{Mn}$ trimer and compares reasonably well with experiments.

\section{ACKNOWLEDGMENTS}

This work is sponsored by the Irish Research Council for Science, Engineering \& Technology (IRCSET). N.B. and S.S. thank Science Foundation of Ireland (Grant No. 08/ERA/I1759) and CRANN for financial support. Computational resources have been provided by the Trinity Centre for High Performance Computing (TCHPC). We wish to thank Cyrus Hirjibehedin for making the experimental data shown in Fig. 7 available to us.

\section{APPENDIX}

Here, we wish to derive a method for calculating the steadystate nonequilibrium distribution of the spin energy levels population $P_{m}(V)$ due to the coupling with the electrodes. In order to do so, we expand Eq. (4) up to the $n$th order in the interaction Hamiltonian:

$$
\begin{aligned}
& {\left[D\left(\tau, \tau^{\prime}\right)\right]_{n m}} \\
& =\sum_{n} \frac{(-i)^{n+1}}{n !} \int_{C} d \tau_{1} \cdots \int_{C} d \tau_{n} \\
& \quad \times \frac{\left\langle 0\left|T_{C}\left\{H_{e-s p}\left(\tau_{1}\right) \cdots H_{e-s p}\left(\tau_{n}\right) d_{n}(\tau) d_{m}^{\dagger}\left(\tau^{\prime}\right)\right\}\right| 0\right\rangle}{U(-\infty,-\infty)},
\end{aligned}
$$

where $U$ is the time-evolution unitary operator and the timeaverages are over the known noninteracting $\left(J_{s d}=0\right)$ ground state $|0\rangle$. As in Eq. (9), the time integration over $\tau$ is ordered on the contour $C$ going from $-\infty$ to $+\infty$ and then returning from $+\infty$ to $-\infty .38$

By inserting the expression for $H_{e-s p}$ from Eq. (2) into the equation above and by expanding up to the second order, we obtain [note for the ease of the description we omit the elastic contribution of $\varepsilon_{0}$, which is then included in the final expression in Eq. (A6)

$$
\begin{aligned}
{\left[D\left(\tau, \tau^{\prime}\right)\right]_{n m}^{(2)}=} & \frac{(-i)^{3}}{2 !} J_{s d}^{2} \sum_{\alpha, \alpha^{\prime}, \beta, \beta^{\prime}} \int_{C} d \tau_{1} \int_{C} d \tau_{2}\left\langle 0\left|T_{C}\left\{c_{\alpha}^{\dagger}\left(\tau_{1}\right) c_{\alpha^{\prime}}\left(\tau_{1}\right) c_{\beta}^{\dagger}\left(\tau_{2}\right) c_{\beta^{\prime}}\left(\tau_{2}\right) d_{n}(\tau) d_{m}^{\dagger}\left(\tau^{\prime}\right)\right\}\right| 0\right\rangle \\
& \times \sum_{i, j}\left\langle 0\left|T_{C}\left\{S^{i}\left(\tau_{1}\right) S^{j}\left(\tau_{2}\right)\right\}\right| 0\right\rangle\left[\sigma^{i}\right]_{\alpha \alpha^{\prime}}\left[\sigma^{j}\right]_{\beta \beta^{\prime}}
\end{aligned}
$$

where the indices $i$ and $j$ run over the cartesian coordinates $x, y$, and $z$ for the given spin coupled to the tip (the tip make electronic contact with one spin only). We now substitute into Eq. (A2) the operator breakdown of the spin from Eq. (5):

$$
\begin{aligned}
{\left[D\left(\tau, \tau^{\prime}\right)\right]_{n m}^{(2)}=} & \frac{(-i)^{3}}{2 !} J_{s d}^{2} \sum_{k, k^{\prime}, l, l^{\prime}} \int_{C} d \tau_{1} \int_{C} d \tau_{2}\left\langle 0\left|T_{C}\left\{d_{n}(\tau) d_{k}^{\dagger}\left(\tau_{1}\right) d_{k^{\prime}}\left(\tau_{1}\right) d_{l}^{\dagger}\left(\tau_{2}\right) d_{l^{\prime}}\left(\tau_{2}\right) d_{m}^{\dagger}\left(\tau^{\prime}\right)\right\}\right| 0\right\rangle \\
& \times \sum_{\alpha, \alpha^{\prime}, \beta, \beta^{\prime}}\left\langle 0\left|T_{C}\left\{c_{\alpha^{\prime}}\left(\tau_{1}\right) c_{\beta}^{\dagger}\left(\tau_{2}\right) c_{\beta^{\prime}}\left(\tau_{2}\right) c_{\alpha}^{\dagger}\left(\tau_{1}\right)\right\}\right| 0\right\rangle \times \sum_{i, j} S_{k k^{\prime}}^{i} S_{l l^{\prime}}^{j}\left[\sigma^{i}\right]_{\alpha \alpha^{\prime}}\left[\sigma^{j}\right]_{\beta \beta^{\prime}} .
\end{aligned}
$$

The time-ordered contractions of the two brackets in Eq. (A3) can be rewritten in terms of their respective noninteracting Green's functions $D_{0}\left(\tau, \tau^{\prime}\right)$ and $G_{0}\left(\tau, \tau^{\prime}\right)$ as follows:

$$
\begin{aligned}
{\left[D\left(\tau, \tau^{\prime}\right)\right]_{n m}^{(2)}=} & -J_{s d}^{2} \sum_{k, k^{\prime}, l, l^{\prime}} \int_{C} d \tau_{1} \int_{C} d \tau_{2} \delta_{n k} \delta_{l k^{\prime}} \delta_{m l^{\prime}}\left[D_{0}\left(\tau, \tau_{1}\right)\right]_{n n}\left[D_{0}\left(\tau_{1}, \tau_{2}\right)\right]_{l l}\left[D_{0}\left(\tau_{2}, \tau^{\prime}\right)\right]_{m m} \\
& \times \sum_{\alpha, \alpha^{\prime}, \beta, \beta^{\prime}} \delta_{\alpha^{\prime} \beta} \delta_{\alpha \beta^{\prime}}\left[G_{0}\left(\tau_{1}, \tau_{2}\right)\right]_{\beta \beta}\left[G_{0}\left(\tau_{2}, \tau_{1}\right)\right]_{\alpha \alpha} \sum_{i, j} S_{k k^{\prime}}^{i} S_{l l^{\prime}}^{j}\left[\sigma^{i}\right]_{\alpha \alpha^{\prime}}\left[\sigma^{j}\right]_{\beta \beta^{\prime}},
\end{aligned}
$$

where the extra factor of 2 emerges from the fact that a second contraction of the time-ordered bracket merely exchanges $\tau_{1}$ and $\tau_{2}$. Then, by using Dyson's equation, ${ }^{39}$ one can write the second-order contribution to the interacting spin self-energy ( $\left.\Pi\right)$. This 
reads

$$
\left[\Pi\left(\tau_{1}, \tau_{2}\right)\right]_{n m}^{(2)}=-2 J_{s d}^{2} \sum_{\alpha, \beta}\left[G_{0}\left(\tau_{1}, \tau_{2}\right)\right]_{\beta \beta}\left[G_{0}\left(\tau_{2}, \tau_{1}\right)\right]_{\alpha \alpha} \sum_{l}\left[D_{0}\left(\tau_{1}, \tau_{2}\right)\right]_{l, l} \sum_{i, j} S_{n l}^{i} S_{l m}^{j}\left[\sigma^{i}\right]_{\alpha \beta}\left[\sigma^{j}\right]_{\beta \alpha}
$$

where we have evoked the assumption that the electrons are spin degenerate thus omitting the spin index on $G_{0}\left(\tau_{1}, \tau_{2}\right)$ and including a factor of 2. We now calculate the real-time quantities, such as the lesser (greater) self-energies, by using Langreth's theorem for the time ordering over the defined contour. ${ }^{38}$ After including the elastic contribution, we obtain

$$
\left[\Pi^{\lessgtr}\left(t_{1}, t_{2}\right)\right]_{n m}^{(2)}=-2 J_{s d}^{2} \sum_{\alpha, \beta}\left[G_{0}^{\lessgtr}\left(t_{1}, t_{2}\right)\right]_{\beta \beta}\left[G_{0}^{\gtrless}\left(t_{2}, t_{1}\right)\right]_{\alpha \alpha} \sum_{l}\left[D_{0}^{\lessgtr}\left(t_{1}, t_{2}\right)\right]_{l l} \sum_{i, j}\left(S_{n l}^{i} S_{l m}^{j}\left[\sigma^{i}\right]_{\alpha \beta}\left[\sigma^{j}\right]_{\beta \alpha}+\delta_{i j} \delta_{\alpha \beta} \chi S_{n m}^{i}\left[\sigma^{i}\right]_{\alpha \beta}\right) \text {. }
$$

On computing the Fourier transform, we note the two different expressions for the lesser and greater Green's functions are

$$
\left[\Pi^{\lessgtr}(E)\right]_{n m}^{(2)}=-\frac{J_{s d}^{2}}{\pi} \sum_{l} P_{l}^{\lessgtr} \sum_{\alpha, \beta} \int_{-\infty}^{+\infty} d \omega\left[G_{0}^{<}(\omega)\right]_{\beta \beta}\left[G_{0}^{>}\left(\omega \pm\left(E-\varepsilon_{l}\right)\right)\right]_{\alpha \alpha} \sum_{i, j}\left(S_{n l}^{i} S_{l m}^{j}\left[\sigma^{i}\right]_{\alpha \beta}\left[\sigma^{j}\right]_{\beta \alpha}+\delta_{i j} \delta_{\alpha \beta} \chi S_{n m}^{i}\left[\sigma^{i}\right]_{\alpha \beta}\right),
$$

where we have defined $P_{l}^{<}=P_{l}$ and $P_{l}^{>}=1-P_{l}$ and $D_{0}^{\lessgtr}\left(t_{1}, t_{2}\right)_{l l}=P_{l}^{\lessgtr} \exp \left[-i \varepsilon_{l}\left(t_{1}-t_{2}\right) / \hbar\right]$. By assuming that the spin system is in thermal contact with a heat bath kept at the temperature $T$, the energy levels $\varepsilon_{l}$ should be broadened by the factor $\beta=k_{\mathrm{B}} T$. This can be neglected for the ease of the calculation since in general $T \ll 1$. However, we do not disregard the broadening in the electronic Green's function due to contact to tip and substrate as this is pivotal to the calculation of the nonequilibrium spin populations.

${ }^{1}$ A. J. Heinrich, J. A. Gupta, C. P. Lutz, and D. M. Eigler, Science 306, 466 (2004).

${ }^{2}$ D. M. Eigler and E. K. Schweizer, Nature (London) 344, 524 (1990). ${ }^{3}$ C. F. Hirjibehedin, C. Lin, A. F. Otte, M. Ternes, C. P. Lutz, B. A. Jones, and A. J. Heinrich, Science 317, 1199 (2007).

${ }^{4}$ S. Loth, C. P. Lutz, and A. J. Heinrich, Nature Physics 6, 340 (2010).

${ }^{5}$ A. F. Otte et al., Nat. Phys. 4, 847 (2008).

${ }^{6}$ J. J. Parks et al., Science 328, 1370 (2010).

${ }^{7}$ C. F. Hirjibehedin, C. P. Lutz, and A. J. Heinrich, Science 312, 1021 (2006).

${ }^{8}$ A. F. Otte, M. Ternes, S. Loth, C. P. Lutz, C. F. Hirjibehedin, and A. J. Heinrich, Phys. Rev. Lett. 103, 107203 (2009).

${ }^{9}$ S. Loth, C. P. Lutz, and A. J. Heinrich, New J. Phys. 12, 125021 (2010).

${ }^{10}$ X. Chen, Y.-S. Fu, S.-H. Ji, T. Zhang, P. Cheng, X.-C. Ma, X.-L. Zou, W.-H. Duan, J.-F. Jia, and Q.-K. Xue, Phys. Rev. Lett. 101, 197208 (2008).

${ }^{11}$ C. Romeike, M. R. Wegewijs, and H. Schoeller, Phys. Rev. Lett. 96, 196805 (2006).

${ }^{12}$ J. Tersoff and D. R. Hamann, Phys. Rev. Lett. 50, 1998 (1983).

${ }^{13}$ J. Fernandez-Rossier, Phys. Rev. Lett. 102, 256802 (2009).

${ }^{14}$ N. Lorente and J. P. Gauyacq, Phys. Rev. Lett. 103, 176601 (2009).

${ }^{15}$ M. Persson, Phys. Rev. Lett. 103, 050801 (2009).

${ }^{16}$ A. Hurley, N. Baadji, and S. Sanvito, Phys. Rev. B 84, 035427 (2011).

${ }^{17}$ A. Hurley, N. Baadji, and S. Sanvito, Phys. Rev. B 84, 115435 (2011).

${ }^{18}$ F. Elste and C. Timm, Phys. Rev. B 81, 024421 (2010).

${ }^{19}$ R. Zitko, R. Peters, and T. Pruschke, New J. Phys. 11, 053003 (2009).

${ }^{20}$ R. Zitko and T. Pruschke, New J. Phys. 12, 063040 (2010).

${ }^{21}$ J. Fransson, O. Eriksson, and A. V. Balatsky, Phys. Rev. B 81, 115454 (2010).

${ }^{22}$ F. Delgado, J. J. Palacios, and J. Fernandez-Rossier, Phys. Rev. Lett. 104, 026601 (2010).
${ }^{23}$ F. Delgado and J. Fernandez-Rossier, Phys. Rev. B 82, 134414 (2010).

${ }^{24}$ F. D. Novaes, N. Lorente, and J. P. Gauyacq, Phys. Rev. B 82, 155401 (2010)

${ }^{25}$ B. Sothmann and J. König, New J. Phys. 12, 083028 (2010).

${ }^{26}$ F. Delgado and J. Fernandez-Rossier, Phys. Rev. B 84, 045439 (2011).

${ }^{27}$ J. Paaske, A. Andersen, and K. Flensberg, Phys. Rev. B 82, 081309(R) (2010).

${ }^{28}$ M. Pletyukhov and D. Schuricht, Phys. Rev. B 84, 041309(R) (2011).

${ }^{29}$ L. V. Keldysh, Sov. Phys. JETP 20, 1018 (1965).

${ }^{30}$ S. Datta, Electronic Transport in Mesoscopic Systems (Cambridge University Press, Cambridge, 1995).

${ }^{31}$ A. R. Rocha, V. M. Garcia-Suarez, S. Bailey, C. Lambert, J. Ferrer, and S. Sanvito, Phys. Rev. B 73, 085414 (2006).

${ }^{32}$ I. Rungger and S. Sanvito, Phys. Rev. B 78, 035407 (2008).

${ }^{33}$ P. Hyldgaard, S. Hershfield, J. H. Davies, and J. W. Wilkins, Ann. Phys. 236, 1 (1994).

${ }^{34}$ W. Lee, N. Jean, and S. Sanvito, Phys. Rev. B 79, 085120 (2009).

${ }^{35} \mathrm{~K}$. Yosida, Theory of Magnetism (Springer-Verlag, Berlin, 1996).

${ }^{36}$ M. Stamenova, T. N. Todorov, and S. Sanvito, Phys. Rev. B 77, 054439 (2008).

${ }^{37}$ A. Pertsova, M. Stamenova, and S. Sanvito, Phys. Rev. B 84, 155436 (2011).

${ }^{38}$ H. Haug and A. P. Jauho, Quantum Kinetics in Transport and Optics of Semiconductors (Springer, Berlin, Heidelberg, 1996).

${ }^{39}$ G. D. Mahan, Many-Particle Physics, 2nd ed. (Plenum, New York, 1990).

${ }^{40}$ P. Lucignano, R. Mazzarello, A. Smogunov, M. Fabrizio, and E. Tosatti, Nat. Mater. 8, 563 (2009).

${ }^{41}$ U. Fano, Phys. Rev. 124, 1866 (1961).

${ }^{42}$ O. Újsághy, J. Kroha, L. Szunyogh, and A. Zawadowski, Phys. Rev. Lett. 85, 2557 (2000). 